Revista de Matemática: Teoría y Aplicaciones 2(1): 57-68 (1995)

\title{
SERIES DE FOURIER APLICADAS A PROBLEMAS DE CÁlCULO DE VARIACIONES CON RETARDO
}

\author{
LOREna SAlazar Solórzano ${ }^{1}$
}

\section{Resumen}

En este artículo presentamos una aproximación de la función que minimiza el funcional

$$
J[X]=\int_{0}^{T} F(t, X(t), X(t-\tau), \dot{X}(t)) d t
$$

aproximando $X(t)$ por medio de expansiones de la serie de Fourier Coseno $X_{n}(t)$. Se dan condiciones bajo las cuales

$$
J\left[X_{n}(t)\right] \longrightarrow J[X(t)] \text { cuando } n \rightarrow \infty .
$$

\footnotetext{
Abstract

In this article we present an approximation of the minimizing function of the functional

$$
J[X]=\int_{0}^{T} F(t, X(t), X(t-\tau), \dot{X}(t)) d t
$$

by approximating $X(t)$ with Cosine Fourier series expansions $X_{n}(t)$. We give conditions under which

$$
J\left[X_{n}(t)\right] \longrightarrow J[X(t)] \quad \text { as } \quad n \rightarrow \infty
$$
}

\section{Introducción}

El problema que se trata de resolver aquí es un problema en cálculo de variaciones con retardo. Más específicamente, se intenta minimizar el funcional:

$$
J[X]=\int_{0}^{T} F[t, X(t), X(t-\tau), \dot{X}(t)] d t,
$$

entre todas las funciones $X(t) \in D$, donde $D$ es el conjunto de las funciones admisibles definidas por

$$
\begin{aligned}
& D=\{X:[-\tau, T] \rightarrow R: X(t)=\phi(t),-\tau \leq t \leq 0 \\
& \left.X \in \mathrm{PC}^{1}[0, T], \quad \text { y } \quad X(T)=B, \quad B \quad \text { constante }\right\}
\end{aligned}
$$

\footnotetext{
${ }^{1}$ Escuela de Matemática, Universidad de Costa Rica
} 
Aquí $\mathrm{PC}^{1}[0, T]$ denota el conjunto de las funciones continuas en $[0, T]$ con derivadas continuas a trozos, y $\phi$ es una función dada continua a trozos en $[-\tau, 0]$. Asumimos además que la función

$$
F:[0, T] \times \mathbb{R} \times \mathbb{R} \times \mathbb{R} \rightarrow \mathbb{R}
$$

es continua y posee derivadas parciales $\frac{\partial F}{\partial X}$ y $\frac{\partial F}{\partial X}$ continuas.

Dado que la mayoría de los fenómenos no ocurren instantáneamente, resulta más apropiado permitir que las variables dependan no sólo del momento actual, sino también de su pasado. En otras palabras, es más natural permitir un retardo en el tiempo. Así, en lugar de tener como condición inicial un sólo punto, la condición más natural es tener una función inicial $\phi$ que refleje la historia del fenómeno en cierto intervalo $[-\tau, 0]$. Esta es una situación más realista en diferentes dominios de aplicación como en física, ingenería, biología, medicina, economía, etc. Así, se trata de buscar una extensión de $\phi(t)$ en el futuro hacia una función $X(t)$ la cual satisfaga el problema anterior. Con el fin de tener continuidad necesitamos que $X(0)=\phi(0)$.

Este tipo de problemas pueden ser formulados de diferentes maneras. Uno de los métodos clásicos para resolver un problema de cálculo de variaciones sin retardo, consiste en resolver la ecuación diferencial de Euler-Lagrange asociada al problema. D. Hughes [5], presenta la ecuación diferencial de Euler-Lagrange análoga para el caso en que existe retardo. Esta es:

$$
\begin{aligned}
& \frac{d}{d t}\left(F_{\dot{X}}[t, X(t), X(t-\tau), \dot{X}(t)]\right)= \\
& \left\{\begin{array}{l}
F_{X}[t, X(t), X(t-\tau), \dot{X}(t)]+F_{X}[t+\tau, X(t+\tau), X(t), \dot{X}(t+\tau)] \\
\text { en } \quad[0, T-\tau] \\
F_{X}[t, X(t), X(t-\tau), \dot{X}(t)], \quad \text { en } \quad[T-\tau, T]
\end{array}\right.
\end{aligned}
$$

Note que esta ecuación diferencial resulta bastante complicada, pues no sólo tiene un retardo sino que también tiene un adelanto en el tiempo. Tal enfoque no da un buen camino a seguir, dado que para resolver la ecuación se necesitaría información no sólo del pasado sino también del futuro, lo cual no tiene sentido en la práctica.

\section{Aproximación por medio de Series de Fourier}

Para aproximar la solución, se seguirá un camino más directo. Se trata de aproximar la solución óptima, la cual se asume que existe, usando la serie Coseno de Fourier.

\section{Problema original:}

$$
\begin{aligned}
J[X] & =\int_{0}^{T} F(t, X(t), X(t-\tau), \dot{X}(t)) d t \\
X(t) & =\phi(t) \quad t \in[-\tau, 0] \\
X(T) & =B, \quad B \text { constante. }
\end{aligned}
$$




\section{Aproximación del problema:}

$$
\begin{aligned}
J\left[X_{n}\right] & =\int_{0}^{T} F\left(t, X_{n}(t), X_{n}(t-\tau), \dot{X}_{n}(t)\right) d t \\
X_{n}(t) & =\phi(t) \quad t \in[-\tau, 0] \\
X_{n}(T) & =B, \quad B \text { constante, }
\end{aligned}
$$

donde $X_{n}(t)$ es la $n$-ésima suma parcial de la serie Coseno de Fourier $X(t)$, i.e;

$$
X_{n}(t)=\left\{\begin{array}{l}
\frac{a_{0}}{2}+\sum_{k=1}^{n} a_{k} \cos \left(\frac{k t \pi}{T}\right) \quad \text { si } \quad t \geq 0 \\
\phi(t) \quad \text { si } \quad-\tau \leq t \leq 0
\end{array}\right.
$$

satisfaciendo las condiciones:

$$
\begin{aligned}
& X_{n}(0)=\frac{a_{0}}{2}+\sum_{k=1}^{n} a_{k}=\phi(0) \\
& X_{n}(T)=\frac{a_{0}}{2}+\sum_{k=1}^{n}(-1)^{k} a_{k}=B .
\end{aligned}
$$

Dado que es común trabajar con expansiones trigonométricas con un período estándar de $2 \pi$, se hace un cambio de variable de $[0, T]$ a $[0, \pi]$, por medio de:

$$
t=\frac{s T}{\pi}
$$

Entonces, la expansión de la serie Coseno de Fourier de $X(t)$ puede ser escrita como:

$$
X(s) \sim \frac{a_{0}}{2}+\sum_{k=1}^{\infty} a_{k} \cos (k s),
$$

y sólo al final de esta discusión, se volverá a la expansión original $X(t)$ en el intervalo $[0, T]$. Se asumirá también la extensión natural de la función $X(t)$ al intervalo $[-T, 0]$. El objetivo es probar el siguiente resultado:

Si $F$ es continua en todas sus variables, y si $X(t)$ es continua, periódica de periodo $2 \pi$, y con una derivada suave a trozos, entonces la serie de Fourier $X_{n}(t)$ de $X(t)$ satisface

$$
\lim _{n \rightarrow \infty} J\left[X_{n}(t)\right]=J[X(t)]
$$

Se usarán seis conocidos lemas para probar este resultado. Las pruebas de los lemas pueden encontrarse en [12]; sin embargo, aquí los enunciados han sido modificados al caso en que la serie de Fourier incluye sólo los términos con coseno. Pruebas de estos lemas ya modificados pueden encontrarse en [9]. 
Lema 1 Si $u \in[0, \pi]$, entonces:

$$
\frac{1}{2}+\sum_{k=1}^{n} \cos (k u)=\frac{\operatorname{sen}[(n+1 / 2) u]}{2 \operatorname{sen}(u / 2)}
$$

Lema 2 Si $X(t)$ es periódica de período $2 \pi$, y si:

$$
X(t) \sim \frac{a_{0}}{2}+\sum_{k=1}^{\infty} a_{k} \cos (k t)
$$

entonces:

$$
X_{n}(t)=\frac{1}{\pi} \int_{0}^{\pi}[X(t+u)+X(u-t)] \frac{\operatorname{sen}[(n+1 / 2) u]}{2 \operatorname{sen}(u / 2)} d u .
$$

Lema 3 Cualquier función $X(t)$ puede ser expresada como:

$$
X(t)=\frac{2}{\pi} \int_{0}^{\pi} X(t) \frac{\operatorname{sen}[(n+1 / 2) u]}{2 \operatorname{sen}(u / 2)} d u
$$

Lema 4 Si $X(t)$ es continua y periódica con período $2 \pi$ y con una derivada absolutamente integrable, y si $W(u)$ es una función con derivada continua para $\alpha \leq u \leq \beta$, entonces para cualquier $\epsilon>0$ se tiene:

$$
\left|\int_{\alpha}^{\beta} X(t+u) W(u) \operatorname{sen}(m u) d u\right| \leq \epsilon,
$$

para $m>0$ suficientemente grande.

Lema 5 Para cualquier $m$, la integral:

$$
\int_{0}^{u} \frac{\operatorname{sen}(m s)}{2 \operatorname{sen}(s / 2)} d s
$$

es acotada para $0 \leq u \leq \pi$.

Lema $6^{2}$ Si $f(t)$ es una función absolutamente integrable, entonces:

$$
\lim _{m \rightarrow \infty} \int_{a}^{b} f(t) \operatorname{sen}(m t) d t=\lim _{m \rightarrow \infty} \int_{a}^{b} f(t) \cos (m t) d t=0,
$$

donde $m$ no es necesariamente un entero.

Con el uso de estos lemas, se pueden establecer algunos criterios de convergencia para la sucesión de funciones $\left\{X_{n}(t)\right\}_{n=1}^{\infty}$. Para ello se asumen además las siguientes condiciones para las funciones admisibles:

\footnotetext{
${ }^{2}$ Este resultado es usualmente conocido como el lema de Riemann-Lebesgue
} 
(A.1) $X(t)$ es continua y periódica de período $2 \pi$,

(A.2) $\dot{X}(t)$ es absolutamente integrable,

(A.3) $\dot{X}(t)$ es suave a trozos.

Las condiciones (A.1) y (A.2) son suficientes para garantizar que $X_{n}(t)$ converge uniformemente a $X(t)$ en $[0, \pi]$, como se mostrará en el Teorema 1 . La condición (A.3) es agregada con el fin de asegurar la convergencia puntual casi por doquier de $\dot{X}_{n}(t)$ a $\dot{X}(t)$ en $[0, \pi]$. Las pruebas de los siguientes teoremas en el caso en que no hay retardo, pueden encontrarse en diferentes textos como en [12], [3], [6] y [7]. Sin embargo, aquí se hacen adaptaciones a estos resultados, para el caso en que sí existe retardo.

Teorema 1 Si $X(t)$ es continua, periódica de período $2 \pi y \dot{X}(t)$ es absolutamente integrable, entonces su serie de Fourier Coseno $X_{n}(t)$ converge uniformemente a $X(t)$ en $[0, \pi]$.

DEMOSTRACIÓN:

Por los lemas 1 y 2 :

$$
\begin{aligned}
& X_{n}(t)-X(t) \\
& =\frac{1}{\pi} \int_{0}^{\pi}[X(u+t)+X(u-t)-2 X(t)] \frac{\operatorname{sen}(n+1 / 2) u}{2 \operatorname{sen}(u / 2)} d u \\
& =\frac{1}{\pi} \int_{0}^{\pi}[X(u+t)-X(t)] \frac{\operatorname{sen}(m u)}{2 \operatorname{sen}(u / 2)} d u+\frac{1}{\pi} \int_{0}^{\pi}[X(u-t)-X(t)] \frac{\operatorname{sen}(m u)}{2 \operatorname{sen}(u / 2)} d u \\
& =I_{1}+I_{2}
\end{aligned}
$$

donde $m=n+1 / 2$. Se probará el resultado para $I_{1}$. El resultado para $I_{2}$ sigue el mismo argumento cambiando $X(u+t)$ por $X(u-t)$. La prueba se basa en dividir el intervalo $[0, \pi]$ en dos subintervalos $[0, \delta]$ y $[\delta, \pi]$, donde $\delta>0$ es cualquier número real con $0<\delta<$ $\pi$. Luego se subdivide $I_{1}$ en dos subintegrales, digamos $I_{1_{1}}, I_{1_{2}}$, sobre estos subintervalos respectivamente. Se probará que $\left|I_{1}\right|<\epsilon / 2$. Empezamos con el intervalo $[0, \delta]$ :

$$
I_{1_{1}}=\frac{1}{\pi} \int_{0}^{\delta}[X(u+t)-X(t)] \frac{\operatorname{sen}(m u)}{2 \operatorname{sen}(u / 2)} d u
$$

Integrando $I_{1_{1}}$ por partes,

$$
\begin{array}{ll}
z=X(u+t)-X(t) & d z=\dot{X}(t+u) \\
d v=\frac{\operatorname{sen}(m u)}{2 \operatorname{sen}(u / 2)} & v=\int_{0}^{u} \frac{\operatorname{sen}(m s)}{2 \operatorname{sen}(s / 2)} d s
\end{array}
$$

resulta:

$$
\begin{aligned}
I_{1_{1}} & =\left.\frac{1}{\pi}[X(u+t)-X(t)] \int_{0}^{u} \frac{\operatorname{sen}(m s)}{2 \operatorname{sen}(s / 2)} d s\right|_{0} ^{\delta}-\frac{1}{\pi} \int_{0}^{\delta} \dot{X}(u+t) \int_{0}^{u} \frac{\operatorname{sen}(m s)}{2 \operatorname{sen}(s / 2)} d s d u \\
& =\frac{1}{\pi}[X(\delta+t)-X(t)] \int_{0}^{\delta} \frac{\operatorname{sen}(m s)}{2 \operatorname{sen}(s / 2)} d s-\frac{1}{\pi} \int_{0}^{\delta} \dot{X}(u+t) \int_{0}^{u} \frac{\operatorname{sen}(m s)}{2 \operatorname{sen}(s / 2)} d s d u
\end{aligned}
$$


Como $X(t)$ es continua y $2 \pi$-periódica, es acotada. Por lo tanto $X(t)$ es uniformemente continua. Entonces para cada $\eta>0$ existe un $\delta=\delta(\eta)$ tal que si $s, t$ son numeros reales con $|s-t|<\delta$, entonces:

$$
|X(s)-X(t)|<\eta
$$

Por lo tanto para $\epsilon>0$ se puede seleccionar $\delta_{1}>0$, tal que para todo $t>0$ y $0<\delta<\delta_{1}$ :

$$
|X(t+\delta)-X(t)|<\pi \epsilon / 4 M
$$

donde $M>0$ es una cota superior para:

$$
\int_{0}^{u} \frac{\operatorname{sen}(m s)}{2 \operatorname{sen}(s / 2)} d s
$$

la cual existe por el lema 5. Por otro lado, como $\dot{X}(t)$ es absolutamente integrable, se tiene que para $\epsilon>0$ existe un $\delta_{2}>0$ tal que para $0<\delta<\delta_{2}$ se tiene:

$$
\int_{t}^{t+\delta}|\dot{X}(s)| d s \leq \pi \epsilon / 4 M
$$

Si $0<\delta<\min \left\{\delta_{1}, \delta_{2}\right\}$, se tiene:

$$
\begin{aligned}
\left|I_{1_{1}}\right| \leq & \frac{1}{\pi}|X(t+\delta)-X(t)|\left|\int_{0}^{\delta} \frac{\operatorname{sen}(m s)}{2 \operatorname{sen}\left(\frac{s}{2}\right)} d s\right| \\
& +\frac{1}{\pi}\left|\int_{0}^{\delta} \dot{X}(u+t)\left(\int_{0}^{u} \frac{\operatorname{sen}(m s)}{2 \operatorname{sen}\left(\frac{s}{2}\right)} d s\right) d u\right| \\
\leq & \frac{1}{\pi}\left(2 \frac{\pi \epsilon}{4 M}\right) M=\frac{\epsilon}{2}
\end{aligned}
$$

Para el otro intervalo $I_{1_{2}}$ sobre $[\delta, \pi]$, la observación esencial es que:

$$
W(u)=1 / 2 \operatorname{sen}(u / 2)
$$

es continua y tiene derivadas continuas. Entonces se tiene:

$$
\begin{aligned}
\left|I_{1_{2}}\right| & =\mid \frac{1}{\pi} \int_{\delta}^{\pi}\left[X(u+t)-X(t) \frac{\operatorname{sen}(m u)}{2 \operatorname{sen}(u / 2)} d u \mid\right. \\
& \leq\left|\frac{1}{\pi} \int_{\delta}^{\pi} X(u+t) \frac{\operatorname{sen}(m u)}{2 \operatorname{sen}(u / 2)} d u\right|+\left|\frac{1}{\pi} \int_{\delta}^{\pi} X(t) \frac{\operatorname{sen}(m u)}{2 \operatorname{sen}(u / 2)} d u\right| \\
& \leq\left|\frac{1}{\pi} \int_{\delta}^{\pi} X(u+t) W(u) \operatorname{sen}(m u) d u\right|+\left|\frac{1}{\pi} \int_{\delta}^{\pi} X(t) W(u) \operatorname{sen}(m u) d u\right| .
\end{aligned}
$$

Usando el lema 4 , se obtiene para $m=n+1 / 2$ suficientemente grande:

$$
\left|I_{1_{2}}\right| \leq \frac{\epsilon}{2}
$$

por lo que se obtiene que $\left|I_{1}\right|<\epsilon$. Así mismo se prueba para $I_{2}$. Así:

$$
\left|X_{n}(t)-X(t)\right| \leq 2 \epsilon
$$


para todo $t$ y para todo $n$ suficientemente grande, lo que implica que:

$$
X_{n}(t) \longrightarrow X(t) \text { uniformemente cuando } n \rightarrow \infty,
$$

como se quería.

Teorema 2 Si $X(t)$ es continua, periódica de período $2 \pi$ con una derivada absolutamente integrable, entonces la serie de Fourier Coseno de $\dot{X}(t)$ puede ser obtenida de la serie de Fourier de $X(t)$ diferenciando término a término.

DEMOSTRACIÓN:

Por el Teorema 1 , se sabe que $X_{n}(t) \rightarrow X(t)$ uniformemente cuando $n \rightarrow \infty$. Por lo tanto:

$$
X(t)=\frac{a_{0}}{2}+\sum_{k=1}^{\infty} a_{k} \cos (k t) .
$$

Sean $A_{k}^{\prime}, B_{k}^{\prime}$ los coeficientes de la serie de Fourier de $\dot{X}(t)$, i.e:

$$
\left.\dot{X}(t) \sim \frac{A_{0}^{\prime}}{2}+\sum_{k=1}^{n}\left\{A_{k}^{\prime} \cos (k t)+B_{k}^{\prime} \operatorname{sen}(k t)\right)\right\} .
$$

Entonces por definición:

$$
\begin{aligned}
A_{0}^{\prime} & =\frac{1}{\pi} \int_{-\pi}^{\pi} \dot{X}(t) d t=\frac{1}{\pi}[X(\pi)-X(-\pi)] \\
& =\frac{1}{\pi}\left(\frac{a_{0}}{2}+\sum_{k=1}^{\infty} a_{k}(-1)^{k}\right)-\frac{1}{\pi}\left(\frac{a_{0}}{2}+\sum_{k=1}^{\infty} a_{k}(-1)^{k}\right) \\
& =0,
\end{aligned}
$$

y

$$
\begin{aligned}
A_{k}^{\prime} & =\frac{1}{\pi} \int_{-\pi}^{\pi} \dot{X}(t) \cos (k t) d t \\
& =\left.\frac{-k}{2 \pi} X(t) \operatorname{sen}(k t)\right|_{-\pi} ^{\pi}+\frac{k}{\pi} \int_{-\pi}^{\pi} X(t) \operatorname{sen}(k t) d t \\
& =0+k\left(\frac{1}{\pi} \int_{-\pi}^{\pi} X(t) \operatorname{sen}(n k t) d t\right) .
\end{aligned}
$$

Como solo estamos considerando la serie de Fourier Coseno de $X(t)$, los términos con seno son cero; es decir los coeficientes $b_{k}=\int_{-\pi}^{\pi} X(t) \operatorname{sen}(k t) d t=0$, entonces:

$$
A_{k}^{\prime}=k b_{k}=0 \text {. }
$$

Además,

$$
\begin{aligned}
B_{k}^{\prime} & =\frac{1}{\pi} \int_{-\pi}^{\pi} \dot{X}(t) \operatorname{sen}(k t) d t \\
& =\left.\frac{1}{\pi}[k X(t) \cos (k t)]\right|_{-\pi} ^{\pi}-\frac{k}{\pi} \int_{-\pi}^{\pi} X(t) \cos (k t) d t \\
& =0-k\left(\int_{-\pi}^{\pi} X(t) \cos (k t) d t\right) \\
& =-k a_{k},
\end{aligned}
$$


así:

$$
\dot{X}(t) \sim \sum_{k=1}^{\infty}-k a_{k} \operatorname{sen}(k t)
$$

lo cual coincide con la diferenciación término a término de la serie:

$$
X(t)=\frac{a_{0}}{2}+\sum_{k=1}^{\infty} a_{k} \cos (k t)
$$

El siguiente teorema, también conocido dentro de la teoría de series de Fourier, plantea la convergencia de $\dot{X}_{n}(t)$ hacia $\ddot{X}(t)$.

Teorema 3 Si $X(t)$ es continua, periódica de período $2 \pi$ y $\dot{X}(t)$ es suave a trozos y absolutamente integrable en $[0, \pi]$, entonces la serie de Fourier de $\dot{X}(t)$ converge casi por doquier (a.e.) en $[0, \pi]$.

DEMOSTRACIÓN:

Dado que $\dot{X}(t)$ es suave a trozos en $[0, \pi], \ddot{X}(t)$ existe excepto en un número finito de puntos. Sea $t$ uno de los puntos donde $\ddot{X}(t)$ existe. Se probará que:

$$
\dot{X}_{n}(t) \rightarrow \dot{X}(t) \quad \text { cuando } \quad n \rightarrow \infty .
$$

Por el lema 2:

$$
X_{n}(t)=\frac{1}{\pi} \int_{0}^{\pi}[X(t+u)+X(u-t)] \frac{\operatorname{sen}(n+1 / 2) u}{2 \operatorname{sen}(u / 2)} d u
$$

Como $\dot{X}(t)$ puede ser obtenida de $X(t)$ diferenciando término a término, se tiene:

$$
\dot{X}_{n}(t)=\frac{1}{\pi} \int_{0}^{\pi}[\dot{X}(t+u)-\dot{X}(u-t)] \frac{\operatorname{sen}(n+1 / 2) u}{2 \operatorname{sen}(u / 2)} d u .
$$

También por el lema 3:

$$
\dot{X}(t)=\frac{2}{\pi} \int_{0}^{\pi} \dot{X}(t) \frac{\operatorname{sen}(n+1 / 2) u}{2 \operatorname{sen}(u / 2)} d u .
$$

Entonces:

$$
\begin{aligned}
& \dot{X}_{n}(t)-\dot{X}(t) \\
& \quad=\frac{1}{\pi} \int_{0}^{\pi}[\dot{X}(t+u)-\dot{X}(u-t)-2 \dot{X}(t)] \frac{\operatorname{sen}(n+1 / 2) u}{2 \operatorname{sen}(u / 2)} d u \\
& =\frac{1}{\pi} \int_{0}^{\pi}([\dot{X}(t+u)-\dot{X}(t)]+[\dot{X}(u-t)-\dot{X}(t)]) \frac{\operatorname{sen}(n+1 / 2) u}{2 \operatorname{sen}(u / 2)} d u \\
& \quad=\frac{1}{\pi} \int_{0}^{\pi}\left(\frac{\dot{X}(t+u)-\dot{X}(t)}{u}+\frac{\dot{X}(u-t)-\dot{X}(t)}{u}\right) u \frac{\operatorname{sen}(n+1 / 2) u}{2 \operatorname{sen}(u / 2)} d u .
\end{aligned}
$$


Dado que $\dot{X}$ es suave a trozos, también lo son $\dot{X}(t+u)-\dot{X}(t)$ y $\dot{X}(u-t)-\dot{X}(t)$ como funciones de $u$. Dividiendo entre $u$, ellas siguen siendo suaves a trozos, dado que la única posible dificultad es que en $u=0$ este cociente no tendiera a un límite finito. Pero:

$$
\begin{aligned}
& \lim _{u \rightarrow 0} \frac{\dot{X}(t+u)-\dot{X}(t)}{u}=\ddot{X}(t) \\
& \lim _{u \rightarrow 0} \frac{\dot{X}(u-t)-\dot{X}(t)}{u}=\ddot{X}(-t)
\end{aligned}
$$

son finitos dado que se asumió que $\ddot{X}(t)$ existe. Por otro lado, se ve que:

$$
\lim _{u \rightarrow 0} \frac{u}{2 \operatorname{sen}(u / 2)}=1<\infty
$$

Por lo tanto, si se define:

$$
W(u)=\left[\frac{\dot{X}(t+u)-\dot{X}(t)}{u}+\frac{\dot{X}(u-t)-\dot{X}(t)}{u}\right] \frac{u}{2 \operatorname{sen}(u / 2)},
$$

se tiene que $W(u)$ es absolutamente integrable, por ser el producto de una función absolutamente integrable con una función acotada. Entonces para $n$ suficientemente grande,

$$
\begin{aligned}
& \dot{X}_{n}(t)-\dot{X}(t) \\
& \left.\quad=\frac{1}{\pi} \int_{0}^{\pi} W(u) \operatorname{sen}(n+1 / 2) u\right) d u \\
& \quad=\frac{1}{\pi} \int_{0}^{\pi} W(u) \operatorname{sen}(n u) \cos (1 / 2) d u+\frac{1}{\pi} \int_{0}^{\pi} W(u) \operatorname{sen}(1 / 2) \cos (n u) d u \\
& \leq \epsilon,
\end{aligned}
$$

por el lema 6 (Riemman-Lebesgue). Entonces $\dot{X}_{n}(t) \rightarrow \dot{X}(t)$ cuando $n \rightarrow \infty$ para casi todo $t \in[0, \pi]$, como se quería.

Hasta ahora se ha probado que si $X(t)$ es continua, periódica, con derivada $\dot{X}(t)$ suave a trozos y absolutamente integrable, entonces

$$
\begin{aligned}
X_{n}(t) & =\frac{a_{0}}{2}+\sum_{k=1}^{n} a_{k} \cos \left(\frac{k t \pi}{T}\right) \longrightarrow X(t) \quad \text { uniformemente. } \\
X_{n}(t-\tau) & =\frac{a_{0}}{2}+\sum_{k=1}^{n} a_{k} \cos \left(\frac{k(t-\tau) \pi}{T}\right) \longrightarrow X(t-\tau) \quad \text { uniformemente. } \\
\dot{X}_{n}(t) & =\sum_{k=1}^{n}-k a_{k} \operatorname{sen}\left(\frac{k t \pi}{T}\right) \longrightarrow \dot{X}(t) \quad \text { a.e. }
\end{aligned}
$$

para $n \rightarrow \infty$. Nótese que se hizo el cambio a las variables originales $[0, T]$.

Finalmente se probará el resultado mencionado al comienzo de esta discusión. 
Teorema 4 Si $F$ es continua en todos sus argumentos, y si la función $X(t)$ satisface la condiciones $[A .1],[A .2]$ y $[A .3]$, entonces $J\left[X_{n}\right] \rightarrow J[X]$ cuando $n \rightarrow \infty$, i.e.

$$
\left.\int_{0}^{T} F\left[t, X_{n}(t), X_{n}(t-\tau), \dot{X}_{n}(t)\right] d t \longrightarrow \int_{0}^{T} F[t, X(t), X(t-\tau), \dot{X}(t))\right] d t
$$

DEMOSTRACIÓN:

Sea $F_{n}=F\left(t, X_{n}(t), X_{n}(t-\tau), \dot{X}_{n}(t)\right)$, entonces:

$$
\begin{aligned}
\mid J & {\left[X_{n}\right]-J[X] \mid } \\
& \leq \int_{0}^{T}\left|F_{n}(t)-F(t)\right| d t \\
& =\int_{0}^{T}\left|F\left(t, X_{n}(t), X_{n}(t-\tau), \dot{X}_{n}(t)\right)-F(t, X(t), X(t-\tau), \dot{X}(t))\right| d t .
\end{aligned}
$$

Como $F$ es continua en todos sus argumentos y como:

$$
\begin{aligned}
X_{n}(t) & \longrightarrow X(t) \quad \text { a.e. } \\
X_{n}(t-\tau) & \longrightarrow X(t-\tau) \quad \text { a.e. } \\
\dot{X}_{n}(t) & \longrightarrow \dot{X}(t) \quad \text { a.e. },
\end{aligned}
$$

entonces:

$$
\left.F\left(t, X_{n}(t), X_{n}(t-\tau), \dot{X}_{n}(t)\right) \longrightarrow F\left(t, X(t), X_{(} t-\tau\right), \dot{X}(t)\right) \quad \text { a.e. }
$$

Por otro lado, como $F$ es acotada, existe un $M>0$ tal que:

$$
\left|F\left(t, X_{n}(t), X_{n}(t-\tau), \dot{X}_{n}(t)\right)\right| \leq M
$$

para todo $t \in[0, T]$ y para todo $n$. Así se tiene una sucesión de funciones medibles $\left\{F_{n}\right\}$ tal que $\left|F_{n}\right| \leq M$ para todo $t \in[0, T]$ y tal que $F_{n} \longrightarrow F$ a.e. Por lo tanto, por el Teorema de Convergencia Acotada se tiene que:

$$
\lim _{n \rightarrow \infty} \int_{0}^{T} F_{n}(t) d t=\int_{0}^{T} \lim _{n \rightarrow \infty} F_{n}(t) d t
$$

por lo que:

$$
\begin{aligned}
\lim _{n \rightarrow \infty}\left|J\left[X_{n}\right]-J[X]\right| & \leq \lim _{n \rightarrow \infty} \int_{0}^{T}\left|F_{n}(t)-F(t)\right| d t \\
& =\int_{0}^{T} \lim _{n \rightarrow \infty}\left|F_{n}(t)-F(t)\right| d t \\
& =0
\end{aligned}
$$

lo cual da el resultado que se quería:

$$
J\left[X_{n}(t)\right] \longrightarrow J[X(t)] \quad \text { as } n \rightarrow \infty .
$$




\section{Conclusión}

Los problemas en Cálculo de Variaciones con retardo difieren mucho de los problemas sin retardo. Los métodos comúnmente usados cuando no hay efectos hereditarios, fallan o no son apropiados con la introducción del retardo. Es necesario desarrollar métodos que sean más directos; es decir, métodos que aproximen la solución óptima por medio de la construcción de una sucesión de funciones que converge a la función deseada. El método presentado aquí podría considerarse uno de ellos.

Todos los resultados usados son conocidos y clásicos de la teoría de Fourier. El resultado es un método muy sencillo, una alternativa que podría usarse como una primera aproximación, con hipótesis fáciles de verificar y las cuales son satisfechas por una amplia variedad de funcionales. Además, el método presentado aquí podría ser programado fácilmente. En particular, ésto se logra usando un paquete comercial llamado GAMS [2], como se presenta en $[10]$.

\section{Referencias}

[1] Akhiezer, N. (1962) The Calculus of Variations. Blaisdell Publishing Company, New York, London.

[2] Brooke, A.; Kendrick, D. and Meeraus, A. (1988) GAMS A User's Guide. The Scientific Press.

[3] Davis, H. (1989) Fourier Series and Orthogonal Functions. Dover Publications, New York.

[4] Gelfand, I. M. and Fomin, S.V (1963) Calculus of Variations. Prentice-Hall Inc., New Jersey.

[5] Hughes, D. (1968) "Variational and optimal control problems with delayed argument", Journal of Optimization Theory and Applications, 2(1): 1-15.

[6] Powers, D. (1979) Boundary Value Problems. Academic Press, New York.

[7] Rees, Ch. (1980) Theory and Applications of Fourier Analysis. Marcel Decker Inc., New York.

[8] Rosenblueth, J.F. (1988) "Systems with time delay in the calculus of variations: a variational approach", IMA Journal of Mathematical Control and Information, Vol. 5: $125-145$.

[9] Salazar, L. (1994) Using GAMS to solve Optimization Problems with time Delay. Tesis de Maestría, The University of Toledo, Ohio, Estados Unidos. Julio de 1994.

[10] Salazar, L. (1995) "GAMS: un paquete de optimización usado en cálculo de variaciones". en Memorias IX Simposio Métodos Matemáticos Aplicados a las Ciencias, J. Trejos (ed.), Universidad de Costa Rica-Instituto Tecnológico de Costa Rica, Turrialba. 
[11] Styczen, K. (1988) "Generalized trigonometric approximation of optimal periodic control problems", Int. J. Control 47(2): 459-476.

[12] Tolstov, G. (1976) Fourier Series. Dover Publications, New York.

[13] Weinstock, R. (1974) Calculus of Variations with Applications to Physics and Engineering, Stanford University, Dover Publications, New York. 